\title{
Methylprednisolone-induced Anaphylaxis Diagnosed by Intradermal Skin Test Not Basophil Activation Test: A Case Report
}

Hitomi Amano

Aichi Medical University: Aichi Ika Daigaku

Yoshiro Kitagawa

Nagakute Kitagawa Children's Clinic

Taichiro Muto

Aichi Medical University: Aichi Ika Daigaku

Akihisa Okumura

Aichi Medical University: Aichi Ika Daigaku

Hideyuki Iwayama ( $\sim$ iwahide1976@gmail.com )

Aichi Medical University https://orcid.org/0000-0002-5218-5815

\section{Case report}

Keywords: Methylprednisolone-induced anaphylaxis, Glucocorticoids, Immediate allergy, Succinate ester, Basophil activation test, Intradermal skin test, Apoptosis

Posted Date: December 11th, 2020

DOI: https://doi.org/10.21203/rs.3.rs-124997/v1

License: @ (i) This work is licensed under a Creative Commons Attribution 4.0 International License. Read Full License

Version of Record: A version of this preprint was published at Allergy, Asthma \& Clinical Immunology on July 13th, 2021. See the published version at https://doi.org/10.1186/s13223-021-00570-1. 


\section{Abstract}

\section{Background}

Anaphylaxis is a severe systemic allergic reaction. Glucocorticoids rarely induce anaphylaxis. Determination of allergens includes the in vivo skin prick test (SPT) and intradermal skin test (IDST) and the in vitro basophil activation test (BAT). However, the usefulness of BAT in determining drug allergens has not been adequately studied.

\section{Case presentation}

A 10-year-old boy was admitted to our hospital because of fever and arthralgia for 2 weeks. He had not been treated with glucocorticoids. According to the laboratory tests and imaging studies, he was suspected to have bacterial myositis and was treated with ceftriaxone. However, his symptoms persisted for more than 2 weeks. With a suspicion of autoinflammatory arthritis, we planned methylprednisolone (mPSL) sodium succinate (MPS) during pulse therapy ( $30 \mathrm{mg} / \mathrm{kg}$ ). Fifteen minutes after the injection of MPSL, he had wheezing and generalized wheal formation with decreased oxygenation. The administration of mPSL was discontinued because anaphylaxis was suspected. Thirty minutes after the administration of oxygen and oral olopatadine, the anaphylactic symptoms resolved.

One month after discharge, SPT, IDST, and BAT were performed under the administration of oral prednisolone. The SPTs for MPS, hydrocortisone sodium succinate (HCS) and prednisolone sodium succinate (PSS) were negative. The IDST for MPS was positive. Moreover, the IDSTs for HCS and PSS were positive, whereas those for dexamethasone sodium phosphate and betamethasone sodium phosphate were negative. The BAT for MPS was negative at $1.0 \%$ and $1.9 \%$ after an incubation time of 1 hour and 24 hours, respectively, although the BAT for histamine as positive control was $60.4 \%$ and $18.3 \%$ after an incubation time of 1 hour and 24 hours, respectively. The BATs for HCS and PSS were negative. Therefore, we diagnosed as anaphylaxis secondary to the succinate ester in MPS.

\section{Conclusions}

In this case, IDST was useful for the diagnosis of MPS-induced anaphylaxis, whereas BAT was negative. This highlighted the need to choose the appropriate procedure in the diagnosis of steroid-induced anaphylaxis. The results in our patient suggested that BAT may be considered when IDST and SPT are negative. Further studies are necessary to clarify the diagnostic strategy for steroid-induced anaphylaxis.

\section{Background}

Anaphylaxis is one of the type I allergic reactions that is secondary to excessive immune response to foreign antigens. Immunoglobulin E (IgE) on the surface of basophils binds to allergens and releases physiologically active substances, such as histamine and serotonin. As a result, vasodilation and increased vascular permeability occur, leading to edema and pruritus. Anaphylaxis is a severe and potentially life-threatening systemic allergic reaction involving multiple organs.

Glucocorticoids are hydrophobic particles, and their intravenous forms have succinate ester to make them hydrophilic. Anaphylaxis secondary to glucocorticoids that contain succinate ester has been reported to have a prevalence of approximately $0.3 \%$, which is not rare [1]. The determination of allergens includes in vivo tests like skin prick test (SPT) and intradermal skin test (IDST), and in vitro tests like the allergen-specific IgE test and basophil activation test (BAT). BAT is a flow cytometry-based assay that measures the expression of activation markers on the surface of basophils following stimulation with an allergen [2]. The usefulness of BAT has been well-studied for determination of food allergens, but not for allergies to drugs, such as glucocorticoids [2-5].

In this study, we reported the case of a boy who developed anaphylaxis secondary to intravenous methylprednisolone (mPSL) sodium succinate (MPS) during pulse therapy. The drug allergy was proven by IDST, but BAT was negative. 


\section{Case Presentation}

A 10-year-old boy was admitted to our hospital because of fever and arthralgia for 2 weeks. His past history was unremarkable and had not been treated with MPSL.

Laboratory test showed leukocytosis of $22,400 / \mu \mathrm{L}$ with $88 \%$ segmented neutrophils and elevated C-reactive protein of $9.21 \mathrm{mg} / \mathrm{mL}$. Magnetic resonance imaging of the lower extremities on fat-suppressed T2-weighted images showed high intensity in the right gluteus maximus muscle. He was suspected to have bacterial myositis and was treated with ceftriaxone. However, his symptoms persisted for more than 2 weeks. With a suspicion of autoinflammatory arthritis, we planned mPSL pulse therapy $(30 \mathrm{mg} / \mathrm{kg})$.

Fifteen minutes after the injection of $\mathrm{mPSL}$, he had wheezing and generalized wheal formation with decreased oxygenation. The administration of mPSL was discontinued because anaphylaxis was suspected. Thirty minutes after the administration of oxygen and oral olopatadine, the anaphylactic symptoms resolved. Oral prednisolone (PSL, $2 \mathrm{mg} / \mathrm{kg} / \mathrm{day}$ ) was started for the treatment of arthritis and afforded resolution of fever and arthralgia. He was discharged from the hospital 1 week after the start of oral PSL with no relapse during 8 months of follow-up.

One month after discharge, SPT, IDST, and BAT were performed under the administration of oral PSL. The SPTs for MPS, hydrocortisone sodium succinate (HCS) and PSL sodium succinate (PSS) were negative. The IDST for MPS resulted to a $7 \times$

$7 \mathrm{~mm}$ wheal and a $9 \times 8 \mathrm{~mm}$ erythema, whereas that for normal saline revealed no wheal or erythema. Therefore, the IDST for MPS was judged as positive. Moreover, the IDSTs for HCS and PSS were positive, whereas those for dexamethasone sodium phosphate (DSP) and betamethasone sodium phosphate (BSP) were negative. The BAT for MPS was negative at $1.0 \%$ and $1.9 \%$ after an incubation time of 1 hour and 24 hours, respectively. On the other hand, the BAT for histamine as positive control was $60.4 \%$ and $18.3 \%$ after an incubation time of 1 hour and 24 hours, respectively. The BATs for HCS and PSS were negative. Based on these results, we diagnosed as anaphylaxis secondary to the succinate ester in MPS.

\section{Discussion And Conclusions}

\section{Anaphylaxis to steroids}

Our patient had anaphylaxis induced by MPS during MPSL pulse treatment for autoinflammatory arthritis. Succinate is the common chemical structure among MPS, HCS, and PSS; notably the IDSTs for all three drugs were positive, indicating that the anaphylaxis was attributable to succinate. Anaphylaxis secondary to systemic steroid administration has been reported, and succinate was found to be the cause in most cases [6]. There had been no report on cross-reactivity between succinate and phosphate ester. In our patient, the SPTs and IDSTs were negative for DSP and BSP.

\section{Prick test, intradermal test, and basophil activation test}

In vivo tests, such as SPT and IDST, are generally used for determining the causative drugs in patients with anaphylaxis [7], although BAT has been reported to be useful in proving anaphylaxis secondary to MPS [2]. We searched for case reports on BAT for steroid-induced anaphylaxis in Pubmed and Ichushi-Web, which is the Japanese database of medical literature updated by the Japan Medical Abstracts Society (Table 1) [2-5, 8]. Among the 10 patients, including ours, BAT was positive in 6 patients and negative in 4. IDST was negative in one patient and SPT was negative in five. In the four patients with negative BAT, IDST was positive in two and SPT was positive in three. These facts suggested that a combination of diagnostic tests is necessary to diagnose steroid-induced anaphylaxis. 
Table 1

Case reports of basophil activation test (BAT) for steroid-induced anaphylaxis in the literature.

\begin{tabular}{|c|c|c|c|c|c|c|c|c|c|c|}
\hline No. & Author & Year & Age & Sex & Primary disease & Steroids & BAT & SPT & IDST & $\begin{array}{l}\text { Challenge } \\
\text { test }\end{array}$ \\
\hline 1 & $\begin{array}{l}\text { Ben Said } \\
\text { [2] }\end{array}$ & 2007 & 18 & $\mathrm{~F}$ & $\begin{array}{l}\text { General } \\
\text { anesthesia }\end{array}$ & MPS & positive & positive & positive & ND \\
\hline 2 & $\begin{array}{l}\text { Lehmann } \\
\text { [8] }\end{array}$ & 2008 & 2 & M & Bronchial asthma & PSS & positive & negative & positive & ND \\
\hline 3 & \multirow{4}{*}{$\begin{array}{l}\text { Aranda } \\
\text { [3] }\end{array}$} & \multirow[t]{4}{*}{2010} & 22 & $\mathrm{~F}$ & Dental infection & MPS & positive & positive & ND & ND \\
\hline 4 & & & 32 & $M$ & Parotitis & MPS & negative & positive & ND & ND \\
\hline 5 & & & 46 & $\mathrm{~F}$ & Dental infection & MPS & positive & negative & negative & positive \\
\hline 6 & & & 65 & $\mathrm{~F}$ & Dental surgery & MPS & positive & negative & positive & ND \\
\hline 7 & \multirow[t]{2}{*}{$\begin{array}{l}\text { Walker } \\
{[5]}\end{array}$} & \multirow[t]{2}{*}{2011} & 26 & M & Bee bite & $\begin{array}{l}\text { PSS, } \\
\text { MPS }\end{array}$ & negative & positive & ND & positive \\
\hline 8 & & & 70 & M & Sudden deafness & $\begin{array}{l}\text { PSS, } \\
\text { HCS }\end{array}$ & positive & negative & positive & ND \\
\hline 9 & $\begin{array}{l}\text { Nucera } \\
{[4]}\end{array}$ & 2011 & 45 & $\mathrm{~F}$ & Dialysis surgery & $\mathrm{HCS}$ & negative & positive & positive & ND \\
\hline 10 & Amano & 2020 & 10 & $M$ & $\begin{array}{l}\text { Autoinflammatory } \\
\text { arthritis }\end{array}$ & MPS & negative & negative & positive & ND \\
\hline
\end{tabular}

In our patient, IDST was positive for steroids that contained succinate ester, whereas BAT was negative, regardless of incubation time. This may be explained by the oral steroid use during blood sampling for BAT. PSL could have reduced the sensitivity of basophils to succinate ester. In this patient, the basophils were incubated with mPSL for 1 or 24 hours for the BAT. Glucocorticoids had been reported to induce DNA fragmentation and apoptosis of basophils for several hours through modulation of gene expression [9], which may have reduced the number of basophils in 24 hours. In addition, the rapid inhibitory effect of glucocorticoids on activated basophils through a nongenomic pathway may have reduced basophil activity in 1 hour [10]. Although BAT is known to be safe [7], its diagnostic value in patients with MPS-induced anaphylaxis should be elucidated, especially when the patient is taking steroids.

In conclusion, IDST was useful for the diagnosis of MPS-induced anaphylaxis in this case, whereas BAT was negative. This highlighted the need to choose the appropriate procedure in the diagnosis of steroid-induced anaphylaxis. The results in our patient suggested that BAT may be considered when IDST and SPT are negative. Further studies are necessary to clarify the diagnostic strategy for steroid-induced anaphylaxis.

\section{List Of Abbreviations}

BAT, basophil activation test; BMZ, betamethasone; BSP, betamethasone sodium phosphate; DEX, dexamethasone; DSP, dexamethasone sodium phosphate; HCS, hydrocortisone sodium succinate; IDST, intradermal skin test; IgE, immunoglobulin E; MPS, methylprednisolone sodium succinate; MPSL, methylprednisolone; PSS, prednisolone sodium succinate; SPT, skin prick test

\section{Declarations}

\section{Ethics approval and consent to participate}




\section{Consent for publication}

Written consent to participate and for publication was obtained from the parents.

\section{Availability of data and materials}

The datasets during and/or analysed during the current study are available from the corresponding author on reasonable request.

\section{Competing interests}

The authors declare that they have no conflict of interest.

\section{Funding}

The authors declare that they have no funding for this research.

\section{Authors' contributions}

HA contributed to the design of the research, the interpretation of data for the patient, and was a major contributor in writing the manuscript. YK, TM, HI contributed to the acquisition and analysis of data from the patient. $\mathrm{HI}$ and $\mathrm{AO}$ revised the manuscript critically for important intellectual content. HI contributed to the final approval of the version to be published. All authors read and approved the final manuscript.

\section{Acknowledgements}

The research of HI was supported by JSPS KAKENHI Grant Numbers 16K19676 and 18K15691, The Nitto Foundation, Aichi Medical University “Aikei-Kai” Foundation, The Japanese Society for Pediatric Endocrinology Future Development Grant, and the Yoshiko \& Seizo Foundation.

\section{References}

1. Burgdorff T, Venemalm L, Vogt T, Landthaler M, Stolz W: IgE-mediated anaphylactic reaction induced by succinate ester of methylprednisolone. Ann Allergy Asthma Immunol. 2002; 89:425-8.

2. Ben Said B, Leray V, Nicolas JF, Rozieres A, Berard F: Methylprednisolone-induced anaphylaxis: diagnosis by skin test and basophil activation test. Allergy. 2010;65:531-2.

3. Aranda A, Mayorga C, Ariza A, Doña I, Blanca-Lopez N, Canto G, Blanca M, Torres MJ: IgE-mediated hypersensitivity reactions to methylprednisolone. Allergy. 2010;65:1376-80.

4. Nucera E, Lombardo C, Aruanno A, Colagiovanni A, Buonomo A, de Pasquale T, Pecora V, Sabato V, Rizzi A, Pascolini L et al: 'Empty sella syndrome': a case of a patient with sodium succinate hydrocortisone allergy. Eur J Endocrinol. 2011;164:139-40.

5. Walker Al, Räwer HC, Sieber W, Przybilla B: Immediate-type hypersensitivity to succinylated corticosteroids. Int Arch Allergy Immunol. 2011;155:86-92. 
6. Baeck M, Marot L, Nicolas JF, Pilette C, Tennstedt D, Goossens A: Allergic hypersensitivity to topical and systemic corticosteroids: a review. Allergy. 2009; 64:978-94.

7. Steiner M, Harrer A, Himly M: Basophil Reactivity as Biomarker in Immediate Drug Hypersensitivity Reactions-Potential and Limitations. Front Pharmacol. 2016;7:171.

8. Lehmann S, Ott H: Glucocorticoid hypersensitivity as a rare but potentially fatal side effect of paediatric asthma treatment: a case report. J Med Case Reports. 2008;2:186.

9. Yoshimura C, Miyamasu M, Nagase H, likura M, Yamaguchi M, Kawanami O, Morita Y, Iwata T, Yamamoto K, Hirai K: Glucocorticoids induce basophil apoptosis. J Allergy Clin Immunol. 2001;108:215-20.

10. Yamagata S, Tomita K, Sano H, Itoh Y, Fukai Y, Okimoto N, Watatani N, Inbe S, Miyajima H, Tsukamoto K et al: Nongenomic inhibitory effect of glucocorticoids on activated peripheral blood basophils through suppression of lipid raft formation. Clin Exp Immunol. 2012;170:86-93. 